Homology, Homotopy and Applications, vol.10(1), 2008, pp.413-436

\title{
THE BOUSFIELD LATTICE FOR TRUNCATED POLYNOMIAL ALGEBRAS
}

\author{
W. G. DWYER AND J. H. PALMIERI \\ (communicated by J. Daniel Christensen)
}

\begin{abstract}
The global structure of the unbounded derived category of a truncated polynomial ring on countably many generators is investigated, via its Bousfield lattice. The Bousfield lattice is shown to have cardinality larger than that of the continuum, and objects with large tensor-nilpotence height are constructed.
\end{abstract}

\section{Introduction}

Suppose that $R$ is a commutative ring. In $\mathrm{D}(R)$, the unbounded derived category of $R$, the derived tensor product $-\underset{R}{\stackrel{Q}{\otimes}}-$ is exact, associative, and commutative. Inspired by stable homotopy theory, we make the following definition: given an object $X$ in $\mathrm{D}(R)$, its Bousfield class is

$$
\langle X\rangle=\left\{W \in \mathrm{obD}(R): X \stackrel{L}{\otimes_{R}} W=0\right\} .
$$

We say that objects $X$ and $Y$ are Bousfield equivalent if $\langle X\rangle=\langle Y\rangle$. The Bousfield classes form a lattice $\mathrm{B}(R)$, where the partial ordering is by reverse inclusion. ${ }^{1}$ For example, $\langle 0\rangle$ is the smallest element and $\langle R\rangle$ is the largest one. The study of stable homotopy theory suggests that the structure of this lattice contains useful information about the category $\mathrm{D}(R)$.

For example, a theorem of Neeman [Nee92] says that if $R$ is commutative and noetherian, then the Bousfield lattice of $\mathrm{D}(R)$ is isomorphic to the Boolean algebra of subsets of $\operatorname{Spec}(R)$. The purpose of this paper is to investigate the situation for a non-noetherian ring.

Fix a field $k$ and integers $n_{i} \geqslant 2$ for all $i \geqslant 1$. For any integer $m \geqslant 1$, consider the truncated polynomial algebra

$$
\Lambda_{m}=k\left[x_{1}, x_{2}, \ldots, x_{m}\right] /\left(x_{i}^{n_{i}} \text { for } 1 \leqslant i \leqslant m\right) .
$$

Grade $\Lambda_{m}$ so that it is locally finite and graded connected, and also so that it is

The first author was partially supported by National Science Foundation grant DMS-0354787.

Received February 11, 2008; published on May 30, 2008.

2000 Mathematics Subject Classification: 18E30, 55U35, 13D07, 13D25.

Key words and phrases: Bousfield lattice, derived category, commutative ring.

This article is available at http://intlpress.com/HHA/v10/n1/a18

Copyright (C) 2008, International Press. Permission to copy for private use granted.

${ }^{1}$ We note that it is not clear in general that the Bousfield classes form a set. If $R$ is countable or noetherian, then they do; otherwise, it is unknown. See Question 5.9. 
graded commutative: for example, set $\operatorname{deg} x_{i}=2^{i}$. Since $\operatorname{Spec}\left(\Lambda_{m}\right)$ contains only one element, the ideal consisting of all elements of positive degree, Neeman's theorem says that the Bousfield lattice of $\mathrm{D}\left(\Lambda_{m}\right)$ is not very interesting: it has two elements, $\langle 0\rangle$ and $\left\langle\Lambda_{m}\right\rangle$, and any two nonzero objects of $\mathrm{D}\left(\Lambda_{m}\right)$ are Bousfield equivalent to each other.

This situation gets much more complicated if we remove the noetherian condition. Let

$$
\Lambda=k\left[x_{1}, x_{2}, x_{3}, \ldots\right] /\left(x_{i}^{n_{i}} \text { for all } i\right)
$$

be a truncated polynomial algebra on countably many generators, graded as $\Lambda_{m}$ is. In this paper, we investigate the Bousfield lattice $B(\Lambda)$. For example, we have the following theorem.

Theorem A. The Bousfield lattice $\mathrm{B}(\Lambda)$ of $\mathrm{D}(\Lambda)$ has cardinality at least $2^{2^{\aleph_{0}}}$.

This is proved as Theorem 4.7; see also the discussion at the end of Section 5. The Bousfield lattice has a largest element $\langle\Lambda\rangle$, a smallest element $\langle 0\rangle$, and a unique minimum nonzero element $\langle I(\mathbf{N})\rangle$. We construct a sublattice $\mathbf{m}$ so that given $\langle I(\mathbf{N})\rangle \leqslant\langle X\rangle\langle\langle Y\rangle \leqslant\langle\Lambda\rangle$ in $\mathbf{m}$, there is an uncountable antichain in the interval between $\langle X\rangle$ and $\langle Y\rangle$ in $\mathbf{m}$. The various joins of these elements form a subset of $\mathrm{B}(\Lambda)$ of cardinality $2^{2^{\aleph_{0}}}$.

The minimality of the class $\langle I(\mathbf{N})\rangle$ has some interesting consequences, including a proof of the telescope conjecture for the category $\mathrm{D}(\Lambda)$; see Section 7 .

We also note that if the base field $k$ is countable, then $\Lambda$ is countable, so [DP01, Theorem 1.2] holds: the Bousfield lattice has cardinality at most $2^{2^{\aleph_{0}}}$. Thus we have the following corollary.

Corollary B. If $k$ is countable, then the Bousfield lattice $\mathrm{B}(\Lambda)$ of $\mathrm{D}(\Lambda)$ has cardinality $2^{2^{\aleph_{0}}}$.

The derived category of this ring has other interesting features; for example, we have the following, which is proved below as Theorem 6.1.

Theorem C. For any integer $n \geqslant 1$, there is an object $X_{n}$ in $\mathrm{D}(\Lambda)$ so that the $n$-fold derived tensor product of $X_{n}$ with itself is nonzero, while the $(n+1)$-fold derived tensor product is zero.

In fact, the object $X_{n}$ may be taken to be a $\Lambda$-module.

We point out that the operations $\oplus$ and $\underset{R}{\otimes}$ on $\mathrm{D}(R)$ induce operations on Bousfield classes, and it is easy to check that $\langle X\rangle \oplus\langle Y\rangle$ is the least upper bound, or join, of $\langle X\rangle$ and $\langle Y\rangle$; this holds more generally for a direct sum indexed by any set. The tensor product operation is more complicated. In the noetherian case, one can use Neeman's result to show that $\langle X\rangle \stackrel{\otimes}{R}_{R}^{L}\langle Y\rangle$ is the greatest lower bound, or meet, of $\langle X\rangle$ and $\langle Y\rangle$, but Theorem $\mathrm{C}$ shows that this need not be true in the non-noetherian case.

We also have a nilpotence theorem (Theorem 8.2), a thick subcategory theorem (Corollary 8.3), and a periodicity theorem (Proposition 8.4), à la Hopkins and Smith [HS98, Hop87]. 


\subsection{Philosophy}

A key idea behind this work is that the derived category $\mathrm{D}(R)$ of a commutative ring $R$ is a stable homotopy category in the sense of [HPS97], and so shares formal properties with the stable homotopy category of spectra. One can take this in two different directions: one can take results (or questions) from stable homotopy theory and apply them (or try to answer them) in $\mathrm{D}(R)$. This can produce results which are interesting on their own, in that they shed some light on the structure of the category $\mathrm{D}(R)$ and hence into the ring $R$ and its modules. Theorem $\mathrm{C}$ is an example, as is the nilpotence theorem 8.2.

One can also try to use derived categories as test cases for questions and conjectures about the stable homotopy category of spectra. Neeman [Nee92] has explored the derived category of a commutative noetherian ring $R$; his work gives us a pretty clear picture of what $\mathrm{D}(R)$ looks like through the lens of stable homotopy theory. One might summarize Neeman's results by saying that the prime ideal spectrum of $R$ governs much of the global structure of the derived category. More generally, if one has any stable homotopy category in which "the homotopy groups of the sphere" forms a noetherian ring $R$, then one can investigate the extent to which Neeman's results still hold, which is to say, how similar the given stable homotopy category is to $\mathrm{D}(R)$; this is done, in part, in [HPS97, Chapter 6].

In the usual stable homotopy category of spectra, though, the homotopy groups of the sphere form a non-noetherian ring, so Neeman's work is not as relevant. So part of the motivation for this paper is that, in order to gain insight into spectra, one should study the derived categories of non-noetherian rings from the stable homotopy theoretic point of view. Furthermore, since the prime ideal spectrum of the stable homotopy groups of spheres is pretty small — when working $p$-locally, there are only two prime ideals in $\pi_{*}\left(S^{0}\right)$ - one should study non-noetherian rings with few prime ideals, hence the ring $\Lambda$ under consideration here. One might hope that results about $\mathrm{D}(\Lambda)$ might provide insight into part of the stable homotopy category of spectra, or at least into part of the Bousfield lattice for that category.

The paper [HP99] asks a number of questions about the Bousfield lattice for the category of spectra, but at least some of those questions make sense in any stable homotopy category. So one goal of this paper was to try to answer those questions. This has been somewhat successful. In this paper we settle the "retract conjecture" [HP99, Conjecture 3.12] — we use Theorem C to show in Corollary 6.2 that this fails in $\mathrm{D}(\Lambda)$ - and the "Dichotomy conjecture" [HP99, Conjecture 7.5] we show in Section 7 that this holds in $D(\Lambda)$. Many of the other conjectures deal with specific spectra for which there are no good analogues in $D(\Lambda)$, and so those are not relevant. Of the relevant ones, the main outstanding one is [HP99, Conjecture 9.1]: is every localizing subcategory of $\mathrm{D}(\Lambda)$ a Bousfield class? (Localizing subcategories are defined in Definition 4.2.) Questions involving localizing subcategories seem to be hard; for example, it is not even known whether there is a set of localizing subcategories. It would be nice to have more information about this conjecture in the category $\mathrm{D}(\Lambda)$, or in derived categories in general. We also mention two other questions about the derived category of a commutative ring $R$ : Question 5.8 below asks whether any object in $\mathrm{D}(R)$ is Bousfield equivalent to an $R$-module. Question 5.9 asks whether the Bousfield lattice $\mathrm{B}(R)$ for $\mathrm{D}(R)$ always forms a set. 


\subsection{Organization}

The structure of the paper is as follows: in Section 2, we set up some basic notation for use throughout the rest of the paper; in particular, for any subset $S$ of $\mathbf{N}=\{1,2,3, \ldots\}$, we examine the $\Lambda$-module

$$
\Lambda(S):=k\left[x_{i}: i \in S\right] /\left(x_{i}^{n_{i}}\right)
$$

and its vector space dual $I(S)$. A homotopy theorist might view $\Lambda(S)$ as a sort of Moore spectrum, at least if $n_{i}=2$ for each $i$, while $I(S)$ is its Brown-Comenetz dual. In Section 3, we set up some homological algebra, and we use it to prove that $\Lambda(T) \stackrel{L}{\otimes} I(U)=0$ if and only if $T^{c} \cap U$ is infinite. In Section 4, we use this result to find the relationships among the Bousfield classes for these objects; for example, we show that if $S \subseteq T$, then $\langle\Lambda(S)\rangle \leqslant\langle\Lambda(T)\rangle$ and $\langle I(T)\rangle \leqslant\langle I(S)\rangle$. We use these kinds of computations to prove Theorem A, and we also show that $I(S){\underset{\Lambda}{\otimes}}_{\Lambda}^{L} I(S)=0$ whenever $S$ is infinite.

In Section 5, we examine other objects constructed from $\Lambda(S)$ and $I(T)$, and discuss the sublattice $\mathbf{m}$ discussed above. We prove Theorem $\mathrm{C}$ in Section 6 . We explore the minimality of $\langle I(N)\rangle$, and some related results, in Section 7. Finally, in Section 8 we prove a nilpotence theorem, a thick subcategory theorem, and a periodicity theorem.

We point out that Neeman [Nee00] has considered a similar situation: he studied the Bousfield classes in the derived category of the ring

$$
k\left[x_{2}, x_{3}, x_{4}, \ldots\right] /\left(x_{2}^{2}, x_{3}^{3}, x_{4}^{4}, \ldots\right),
$$

where $k$ is a field. He showed that there were at least $2^{2^{\aleph_{0}}}$ Bousfield classes for this ring as well. His methods were a bit different, and his goal was to demonstrate the size of the Bousfield lattice, not to investigate its finer properties, as we are trying to do here.

\section{Acknowledgements}

Mark Hovey, James Zhang, and Paul Smith have provided valuable input into various aspects of this research.

\section{Notation and basic algebra}

All rings in this paper are graded, as are all modules over them. The suspension or shift functor $\Sigma$ on graded modules is defined as follows: given a graded module $M=\bigoplus_{m} M_{m}$, the module $\Sigma M$ has $m$ th graded piece $M_{m-1}$. Iterating this, $\Sigma^{j} M$ has $m$ th graded piece $M_{m-j}$ for any integer $j$.

All chain complexes in this paper are bigraded, with the first grading the homological one, and the second grading induced by that on the modules. The differential on such a complex will always have degree $(1,0)$. Given integers $i$ and $j$, the $(i, j)$ suspension functor $\Sigma^{i, j}$ is defined as follows: given a graded ring $R$ and a chain complex $X=\left(\cdots \rightarrow X^{n} \rightarrow X^{n+1} \rightarrow \ldots\right)$ of graded $R$-modules, $\Sigma^{i, j} X$ is the chain complex with $n$th term $\Sigma^{j} X^{n-i}$. (Note that $\Sigma$ with a single grading means the shift functor on modules, while with a double grading it is the shift functor on complexes.) 
Fix a field $k$ and a sequence of integers $n_{i} \geqslant 2$ for $i \geqslant 1$. As in (1.1), we define the $k$-algebra $\Lambda$ by

$$
\Lambda=k\left[x_{1}, x_{2}, x_{3}, \ldots\right] /\left(x_{i}^{n_{i}} \text { for all } i\right)
$$

with $\operatorname{deg} x_{i}=2^{i}$. The unbounded derived category $\mathrm{D}(\Lambda)$ is a stable homotopy category, and more precisely a monogenic stable homotopy category; if $k$ is countable, then $D(\Lambda)$ is also a Brown category. See [HPS97, Definitions 1.1.4 and 4.1.4] for the definitions and [HPS97, Section 9.3] for the proof that $D(\Lambda)$ has these properties. From the stable homotopy theoretic point of view, the derived tensor product is the smash product in $\mathrm{D}(\Lambda)$, and the rank one free module $\Lambda$ is the sphere object.

Notation 2.1. Let $\mathbf{N}$ be the set of positive integers and let $S$ be a subset of $\mathbf{N}$. Let $\Lambda(S)$ be the subalgebra of $\Lambda$ generated by $x_{i}$ with $i \in S$; we also write $S^{c}$ for the complement of $S$ in $\mathbf{N}$.

Then for any $S \subseteq \mathbf{N}$ there is an algebra isomorphism $\Lambda \cong \Lambda(S) \otimes \Lambda\left(S^{c}\right),{ }^{2}$ which leads to two algebra homomorphisms: the inclusion map $\Lambda(S) \rightarrow \Lambda$ and the projection $\operatorname{map} \Lambda \rightarrow \Lambda(S)$. These, respectively, lead to two useful functors: the restriction functor $-\left.\right|_{S}: \Lambda$-Mod $\rightarrow \Lambda(S)$-Mod, and the inclusion functor $\iota_{S}: \Lambda(S)$-Mod $\rightarrow \Lambda$-Mod. More generally, if $S \subseteq T \subseteq \mathbf{N}$, then we have functors

$$
\begin{aligned}
-\left.\right|_{S} ^{T}: \Lambda(T)-\operatorname{Mod} & \rightarrow \Lambda(S) \text {-Mod }, \\
\iota_{S}^{T}: \Lambda(S)-\operatorname{Mod} & \rightarrow \Lambda(T) \text {-Mod. }
\end{aligned}
$$

These also induce functors on the corresponding derived categories; we write those as $-\left.\right|_{S} ^{T}$ and $\iota_{S}^{T}$ as well. For any object $X$ in $\mathrm{D}(\Lambda(S))$, we have $\left.\left(\iota_{S}^{T} X\right)\right|_{S} ^{T} \cong X$.

Notation 2.2. Fix $S \subseteq T \subseteq \mathbf{N}$.

(a) We make $\Lambda(S)$ into a $\Lambda(T)$-module by applying the inclusion functor $\iota_{S}^{T}$ to a rank one free $\Lambda(S)$-module; then we have isomorphisms of $\Lambda(T)$-modules

$$
\Lambda(S) \cong \Lambda(T) /\left(x_{i}: i \notin S\right) \cong \Lambda(T) \otimes_{\Lambda(T-S)} k .
$$

Note that this makes $\Lambda(S)$ into a ring object in $\mathrm{D}(\Lambda(T))$.

(b) As a $\Lambda(T)$-module, let $I(S)=\operatorname{Hom}_{k}^{*}(\Lambda(S), k)$ be the graded $k$-dual of $\Lambda(S)$, concentrated in non-positive degrees.

Note that if $S$ is finite, then $I(S)$ is isomorphic to $\Lambda(S)$, up to suspension.

\section{Shapiro's lemma and Brown-Comenetz duality}

In this section, we state Shapiro's lemma for $D(\Lambda)$, we define Brown-Comenetz duality in this setting, and we combine these two concepts to compute some tensor products. Lemma 3.6, which says that $\Lambda(T) \stackrel{L}{\otimes} I(U)=0$ if and only if $T^{c} \cap U$ is infinite, is used throughout the paper.

We start with some basic homological algebra.

\footnotetext{
${ }^{2}$ Any unadorned tensor product in this paper is over the base field $k$.
} 
Lemma 3.1 (Shapiro's lemma). Fix a subset $S$ of $\mathbf{N}$.

(a) [Homology] For any object $X$ in $\mathrm{D}(\Lambda)$, there is an isomorphism

$$
X \stackrel{L}{\otimes} \Lambda\left(S^{c}\right) \cong \iota_{S}\left(\left.X\right|_{S} \underset{\Lambda(S)}{\stackrel{L}{\otimes}} k\right) .
$$

(b) [Cohomology] For any object $X$ in $\mathrm{D}(\Lambda)$, there is an isomorphism

$$
\operatorname{Hom}_{\mathrm{D}(\Lambda)}\left(\Lambda\left(S^{c}\right), X\right)^{*} \cong \operatorname{Hom}_{\mathrm{D}(\Lambda(S))}^{*}\left(k,\left.X\right|_{S}\right) .
$$

Proof. Both parts are standard. For part (a), for example, we have

$$
X \underset{\Lambda}{\otimes} \Lambda\left(S^{c}\right) \cong X \underset{\Lambda}{\stackrel{L}{\otimes}}\left(\left.\Lambda\right|_{S} \underset{\Lambda(S)}{\otimes} k\right) \cong X \underset{\Lambda}{\otimes}\left(\left.\Lambda\right|_{S} \underset{\Lambda(S)}{\stackrel{L}{\otimes}} k\right) \cong \iota_{S}\left(\left.X\right|_{S} \underset{\Lambda(S)}{\stackrel{L}{\otimes}} k\right) .
$$

Part (b) is similar.

Here is one Ext calculation for later use.

Lemma 3.2. Fix $T \subseteq S \subseteq \mathbf{N}$. If $T$ is infinite, then for any set $J$,

$$
\operatorname{Ext}_{\Lambda(S)}^{q}\left(k, \bigoplus_{J} \Lambda(T)\right)=0 \text { for all } q \geqslant 0 .
$$

Proof. We compute the Ext groups in question with the spectral sequence associated to the central algebra extension

$$
k \rightarrow \Lambda(T) \rightarrow \Lambda(S) \rightarrow \Lambda(T-S) \rightarrow k .
$$

For any $\Lambda(S)$-module $N$, there is a strongly convergent spectral sequence [CE56, Theorem XVI.6.1]

$$
\operatorname{Ext}_{\Lambda(S-T)}^{p}\left(k, \operatorname{Ext}_{\Lambda(T)}^{q}(k, N)\right) \Rightarrow \operatorname{Ext}_{\Lambda(S)}^{p+q}(k, N) .
$$

We apply this with $N=\bigoplus \Lambda(T)$. We claim that the groups

$$
\operatorname{Ext}_{\Lambda(T)}^{q}\left(k, \bigoplus_{J} \Lambda(T)\right)
$$

are zero for all $q$.

Since $T$ is infinite, then $\Lambda(T)$ is what Margolis calls a $P$-algebra [Mar83, Chapter 13]. Therefore by [Mar83, Theorem 13.12], any free bounded-below $\Lambda(T)$-module is also injective. In particular, $\bigoplus \Lambda(T)$ is injective, so the above Ext group (3.3) is zero when $q>0$.

Also because $T$ is infinite, there are no $\Lambda(T)$-module maps $k \rightarrow \bigoplus \Lambda(T)$, and we see that the above Ext group (3.3) is zero when $q=0$.

Now we move on to Brown-Comenetz duality. See [BC76] and [Rav84] for some information about this construction in stable homotopy. The analogue here is vector space duality: for any object $Y$ in $\mathrm{D}(\Lambda)$, we define its Brown-Comenetz dual to be the object $I(Y)=\mathrm{RHom}_{k}(Y, k)$ in $\mathrm{D}(\Lambda)$. For example, the Brown-Comenetz dual of $\Lambda(S)$ is $I(\Lambda(S))=I(S)$. 
Lemma 3.4. For any object $X$ in $\mathrm{D}(\Lambda)$, we have

$$
\operatorname{Hom}_{k}^{*}\left(H^{*}(X \stackrel{L}{\otimes} Y), k\right) \cong \operatorname{Hom}_{\mathrm{D}(\Lambda)}^{*}(X, I(Y)) .
$$

Thus if $Y$ is locally finite and $\operatorname{RHom}_{\Lambda}^{*}(X, Y)=0$, then $X \underset{\Lambda}{\stackrel{L}{\otimes}} I(Y)=0$.

Proof. The first statement follows from tensor-hom adjointness: in the category of $\Lambda$-modules, we have

$$
\operatorname{Hom}_{\Lambda}(X, I(Y))=\operatorname{Hom}_{\Lambda}\left(X, \operatorname{Hom}_{k}(Y, k)\right) \cong \operatorname{Hom}_{k}(X \underset{\Lambda}{\otimes} Y, k) .
$$

This induces the above isomorphism in the derived category.

The second statement now follows: since $Y$ is locally finite, it is self-double-dual: $I(I(Y)) \cong Y$. First, $\operatorname{RHom}_{\mathrm{D}(\Lambda)}^{*}(X, Y)=0$ if and only if $\operatorname{Hom}_{\mathrm{D}(\Lambda)}^{*}(X, I(I(Y)))=0$, and then by the first part, we have

$$
\operatorname{Hom}_{k}\left(H^{*}(X \stackrel{L}{\stackrel{\leftrightarrow}{\&}} I(Y)), k\right)=0 .
$$

This implies that $H^{*}(X \underset{\Lambda}{\stackrel{L}{\otimes}} I(Y))=0$, which means that $X \underset{\Lambda}{\stackrel{L}{\otimes}} I(Y)=0$.

Corollary 3.5. In particular, $\operatorname{RHom}_{\Lambda}^{*}(X, \Lambda(S))=0$ if and only if $X \underset{\Lambda}{\stackrel{L}{\otimes}} I(S)=0$.

Now we start computing some tensor products.

Lemma 3.6. Let $T$ and $U$ be subsets of $\mathbf{N}$. Then $\Lambda(T) \underset{\Lambda}{\stackrel{L}{\otimes}} I(U)=0$ if and only if $T^{c} \cap U$ is infinite.

Proof. By Corollary 3.5, we have

$$
\Lambda(T) \stackrel{\perp}{\otimes} I(U)=0 \Longleftrightarrow \operatorname{Hom}_{\mathrm{D}(\Lambda)}^{*}(\Lambda(T), \Lambda(U))=0 .
$$

According to Shapiro's lemma 3.1,

$$
\operatorname{Hom}_{\mathrm{D}(\Lambda)}^{*}(\Lambda(T), \Lambda(U)) \cong \operatorname{Hom}_{\mathrm{D}\left(\Lambda\left(T^{c}\right)\right)}^{*}\left(k,\left.\Lambda(U)\right|_{T^{c}}\right),
$$

and as a $\Lambda\left(T^{c}\right)$-module, $\Lambda(U)$ is isomorphic to

$$
\Lambda\left(U \cap T^{c}\right) \otimes \Lambda(U \cap T) \cong \bigoplus \Lambda\left(U \cap T^{c}\right),
$$

where the direct sum is indexed by a basis for $\Lambda(U \cap T)$, since $\Lambda(U \cap T)$ is trivial as a $\Lambda\left(T^{c}\right)$-module. So we want to understand whether

$$
\operatorname{Hom}_{\mathrm{D}\left(\Lambda\left(T^{c}\right)\right)}^{*}(k, \bigoplus \Lambda(U \cap T))
$$

is zero. If $U \cap T$ is infinite, then Lemma 3.2 shows that this group is zero. If $U \cap T$ is finite, then $\Lambda(U \cap T)$ is a finite-dimensional vector space, and there are $\Lambda\left(T^{c}\right)$-module maps $k \rightarrow \Lambda(U \cap T)$ and thus nonzero maps $k \rightarrow \bigoplus \Lambda(U \cap T)$; thus this group is nonzero. 


\section{Beginning Bousfield class computations}

Now we use Lemma 3.6 to get information about the Bousfield class of $\Lambda(S)$ for subsets $S \subseteq \mathbf{N}$.

We start by constructing some useful exact triangles. Recall that $n_{i}$ is the nilpotence height of the generator $x_{i} \in \Lambda$.

Lemma 4.1. Fix subsets $S \subsetneq T \subseteq \mathbf{N}$ and an integer $i \in T-S$. There are objects $M_{j}, 1 \leqslant j \leqslant n_{i}$, with $M_{1}=\Lambda(S)$ and $M_{n_{i}} \cong \Lambda(S \cup\{i\})$, and $n_{i}-1$ exact triangles in $\mathrm{D}(\Lambda(T))$

$$
\Sigma^{1,0} \Lambda(S) \rightarrow \Sigma^{0, \operatorname{deg} x_{i}} M_{j-1} \rightarrow M_{j}, \quad 2 \leqslant j \leqslant n_{i} .
$$

Proof. There are exact sequences of $\Lambda(i)$-modules

$$
\begin{gathered}
0 \rightarrow \Sigma^{\operatorname{deg} x_{i}} k \rightarrow k\left[x_{i}\right] /\left(x_{i}^{2}\right) \rightarrow k \rightarrow 0, \\
0 \rightarrow \Sigma^{\operatorname{deg} x_{i}} k\left[x_{i}\right] /\left(x_{i}^{2}\right) \rightarrow k\left[x_{i}\right] /\left(x_{i}^{3}\right) \rightarrow k \rightarrow 0, \\
\vdots \\
0 \rightarrow \Sigma^{\operatorname{deg} x_{i}} k\left[x_{i}\right] /\left(x_{i}^{j-1}\right) \rightarrow k\left[x_{i}\right] /\left(x_{i}^{j}\right) \rightarrow k \rightarrow 0, \\
\vdots \\
0 \rightarrow \Sigma^{\operatorname{deg} x_{i}} k\left[x_{i}\right] /\left(x_{i}^{n_{i}-1}\right) \rightarrow \Lambda(i) \rightarrow k \rightarrow 0 .
\end{gathered}
$$

So define $M_{j}$ by

$$
M_{j}=\Lambda(S) \underset{\Lambda(i)}{\stackrel{L}{\otimes}} k\left[x_{i}\right] /\left(x_{i}^{j}\right) .
$$

Then applying $\Lambda(S \cup\{i\}) \otimes_{\Lambda(i)}$ - to these exact sequences gives short exact sequences of $\Lambda(T)$-modules, and hence the desired exact triangles.

\section{Definition 4.2.}

(a) Given subsets $S$ and $T$ of $\mathbf{N}$, we say that $T$ is cofinite in $S$ if $T \subseteq S$ and the complement of $T$ in $S$ is finite. We say that subsets $S$ and $T$ of $\mathbf{N}$ are commensurable if $S \cap T$ is cofinite in both $S$ and $T$. We write $S \sim T$ if $S$ and $T$ are commensurable. We write $S \lesssim T$ if $S$ is commensurable with a subset of $T$.

(b) A full subcategory of $\mathrm{D}(\Lambda)$ is a localizing subcategory if it is triangulated and closed under arbitrary direct sums (and hence arbitrary colimits). For any object $Y$ of $\mathrm{D}(\Lambda)$, the localizing subcategory generated by $Y$ is the intersection of all of the localizing subcategories containing $Y$. Given two objects $X$ and $Y$ of $\mathrm{D}(\Lambda)$, we say that $X$ can be built from $Y$ if $X$ is in the localizing subcategory generated by $Y$.

(c) A subcategory $\mathrm{D}$ of $\mathrm{D}(\Lambda)$ is called thick if it is triangulated and "closed under retracts": if $X \oplus Y$ is in $\mathrm{D}$, then so are $X$ and $Y$. For any object $Y$ of $\mathrm{D}(\Lambda)$, we write thick $(Y)$ for the thick subcategory generated by $Y$, that is, the intersection of all of the thick subcategories containing $Y$.

Note that any localizing subcategory is thick by the Eilenberg swindle [HPS97, Lemma 1.4.9], but thick subcategories need not be localizing. Also, it is well known 
that in the derived category of any commutative ring $R$, every object can be built from $R$; see [HPS97, Theorem 9.3.1], for example.

We recall from the introduction the definition of the Bousfield class $\langle X\rangle$ of an object $X$ :

$$
\langle X\rangle=\{W \in \mathrm{obD}(R): X \underset{R}{\otimes} W=0\} .
$$

These are partially ordered by reverse inclusion.

Lemma 4.3. Fix subsets $S$ and $T$ of $\mathbf{N}$.

(a) $\Lambda(S)$ is in the thick subcategory generated by $\Lambda(T)$ if and only if $T$ is cofinite in $S$.

(b) The following are equivalent:

(1) $S \lesssim T$.

(2) $\Lambda(\widetilde{S})$ can be built from $\Lambda(T)$.

(3) $\langle\Lambda(S)\rangle \leqslant\langle\Lambda(T)\rangle$.

Proof. (a) First assume that $T$ is cofinite in $S$; we want to show that $\Lambda(S)$ is in thick $(\Lambda(T))$. It is enough to assume that $S=T \cup\{i\}$, in which case the exact triangles in Lemma 4.1 do the job.

Now assume that $T$ is not cofinite in $S$. There are two cases to consider: either $T$ is not a subset of $S$, or $T \subseteq S$ and $S-T$ is infinite. Since

$$
\operatorname{Hom}_{\mathrm{D}(\Lambda(T))}^{*}(\Lambda(T), k) \cong \operatorname{Ext}_{\Lambda(T)}^{*}(\Lambda(T), k),
$$

and this is isomorphic to $k$ concentrated in degree 0 , then $\operatorname{Hom}_{\mathrm{D}(\Lambda(T))}^{*}\left(\left.X\right|_{T}, k\right)$ is finite-dimensional for any object $X$ in thick $(\Lambda(T))$. When viewed as a $\Lambda(T)$-module, $\Lambda(S)$ splits as a direct sum:

$$
\left.\left.\left.\left.\Lambda(S)\right|_{T} \cong \Lambda(S-T)\right|_{T} \otimes \Lambda(T \cap S)\right|_{T} \cong \bigoplus \Lambda(T \cap S)\right|_{T}
$$

where the direct sum is indexed by a basis for $\Lambda(S-T)$, since $\Lambda(S-T)$ is trivial as a $\Lambda(T)$-module. If $S-T$ is infinite, then so is this direct sum, and hence so is $\operatorname{Ext}_{\Lambda(T)}^{0}\left(\left.\Lambda(S)\right|_{T}, k\right)$. Therefore $\Lambda(S)$ cannot be in thick $(\Lambda(T))$. Otherwise, Shapiro's lemma 3.1 says that

$$
\operatorname{Ext}_{\Lambda(T)}^{*}(\Lambda(T \cap S), k) \cong \operatorname{Ext}_{\Lambda(T-T \cap S)}^{*}(k, k),
$$

and this is infinite-dimensional as long as $T \neq T \cap S$, and so, again, $\Lambda(S)$ cannot be in thick $(\Lambda(T))$.

(b) To show that $\Lambda(S)$ can be built from $\Lambda(T)$ if $S \lesssim T$, we need to prove that $\Lambda(S)$ can be built from $\Lambda(T)$ if either $S=T \cup\{i\}$ or if $S \subseteq T$. If $S=T \cup\{i\}$, then part (a) shows us that in this case $\Lambda(S)$ is in thick $(\Lambda(T))$ and hence can be built from $\Lambda(T)$.

Now we assume that $S \subseteq T$. First we work in the category $\mathrm{D}(\Lambda(T))$. Every object in $\mathrm{D}(\Lambda(T))$ may be built from $\Lambda(T)$, and in particular $\left.\Lambda(S)\right|_{T}$ may be built from $\Lambda(T)$. Now apply the inclusion functor $\iota_{T}: \mathrm{D}(\Lambda(T)) \rightarrow \mathrm{D}(\Lambda)$ : this functor is the identity on objects, so it is exact and commutes with direct limits; thus in $\mathrm{D}(\Lambda), \Lambda(S)$ may be built from $\Lambda(T)$. This finishes the proof that (1) implies (2). 
(One can make this sort of argument more explicit: if $P_{*}$ is a projective resolution of $\Lambda(S)$ as a $\Lambda(T)$-module, then $\Lambda(S)$ is the colimit of the truncations

$$
0 \rightarrow P_{n} \rightarrow \cdots \rightarrow P_{0} \rightarrow 0
$$

of $P_{*}$. One can then show by a simple induction argument that each of these may be built from $\Lambda(T)$.)

Since the derived tensor product is exact and commutes with direct limits, if $X$ can be built from $Y$, then $\langle X\rangle \leqslant\langle Y\rangle$. Thus (2) implies (3).

Now we prove that (3) implies (1). Assume that $S$ is not commensurable with any subset of $T$; we will show that $\langle\Lambda(S)\rangle$ is not less than or equal to $\langle\Lambda(T)\rangle$. If $S \Varangle T$, then, in particular, $S$ is not commensurable with $S \cap T$, so $S \cap T$ is not cofinite in $S$. If we let $U=S-(S \cap T)$, then we see that $U$ is an infinite subset of $T^{c}$ which does not intersect $S^{c}$. So by Lemma 3.6, we have

$$
I(U) \stackrel{L}{\otimes} \Lambda(T)=0 \quad \text { but } \quad I(U) \underset{\Lambda}{\stackrel{L}{\otimes}} \Lambda(S) \neq 0 .
$$

Thus $\langle\Lambda(T)\rangle$ is not greater than or equal to $\langle\Lambda(S)\rangle$, as desired.

Corollary 4.4. $k=\Lambda(\emptyset)$ may be built from $\Lambda(S)$ for any $S \subseteq \mathbf{N}$.

Corollary 4.5. For subsets $S$ and $T$ of $\mathbf{N},\langle\Lambda(S)\rangle=\langle\Lambda(T)\rangle$ if and only if $S \sim T$. Thus there are uncountably many Bousfield classes in $\mathrm{D}(\Lambda)$. Indeed, there is an uncountable antichain in the Bousfield lattice.

Recall that an antichain in a partially ordered set is a subset any two of whose elements are not comparable.

Proof. For each prime number $p$, let $P_{p}=\left\{p^{k}: k \in N\right\}$. We claim that there is an uncountable set $\left\{S_{\alpha}\right\}$ of subsets of $\mathbf{N}$ such that

(i) the sets $S_{\alpha} \cap P_{p}$ and $S_{\alpha}^{c} \cap P_{p}$ are infinite for all $\alpha, p$, and

(ii) the commensurability classes of $S_{\alpha}$ and $S_{\beta}$ are not comparable, for all $\alpha, \beta$.

First, there are infinitely many such subsets; for instance, for each prime $q$, define $S_{q}$ to be $\bigcup_{p}\left\{p^{k q}: k \in \mathbf{N}\right\}$.

Now suppose that $S_{2}, S_{3}, S_{5}, \ldots$ are any subsets of $N$, indexed by the prime numbers, satisfying (i) and (ii) above. We construct a set $T$, satisfying (i), whose commensurability class is not comparable to that of any $S_{p}$. Let

$$
T=\bigcup_{p}\left(P_{p}-\left(S_{p} \cap P_{p}\right)\right) .
$$

Then for each $p$, the sets $T \cap P_{p}$ and $S_{p} \cap P_{p}$ form a partition of $P_{p}$ into two infinite sets, and hence $T$ is as advertised. Therefore the collection of subsets of $\mathbf{N}$ satisfying (i) and (ii) is not countable.

Finally, given an uncountable set $\left\{S_{\alpha}\right\}$ of subsets of $\mathbf{N}$ satisfying (i) and (ii), the set of Bousfield classes $\left\{\left\langle\Lambda\left(S_{\alpha}\right)\right\rangle\right\}$ is uncountable, and no two elements of it are comparable.

Remark 4.6. The uncountable antichain in the corollary consists of Bousfield classes of $\Lambda\left(S_{\alpha}\right)$ for various subsets $S_{\alpha}$ of $\mathbf{N}$. A simple modification in the proof yields this 
result: for any subsets $T, U$ of $\mathbf{N}$ with $T \lesssim U$ and $T \not U$, there is an uncountable antichain in the interval between $\langle\Lambda(T)\rangle$ and $\langle\Lambda(U)\rangle$ : one may choose each $S_{\alpha}$ so that $T \lesssim S_{\alpha} \lesssim U$

Theorem 4.7. The lattice $\mathrm{B}=\mathrm{B}(\Lambda)$ has cardinality at least $2^{2^{\aleph_{0}}}$.

Proof. By Corollary 4.5, B contains an uncountable antichain; indeed, the elements of the antichain may be chosen to be $\Lambda\left(S_{\alpha}\right)$, where $\left\{S_{\alpha}\right\}_{\alpha \in J}$ is an uncountable set of subsets of $\mathbf{N}$, no two of which have comparable commensurability classes. Here, $J$ is some indexing set with cardinality at least $2^{\aleph_{0}}$. For any subset $I$ of $J$, let

$$
X_{I}=\bigoplus_{\alpha \in I} \Lambda\left(S_{\alpha}\right)
$$

We claim that the objects $X_{I}$ have distinct Bousfield classes: if $I \neq I^{\prime}$, then $\left\langle X_{I}\right\rangle \neq\left\langle X_{I^{\prime}}\right\rangle$. If $I \neq I^{\prime}$, then either $I-I^{\prime}$ or $I^{\prime}-I$ is nonempty; without loss of generality, suppose that $\alpha \in I-I^{\prime}$. Then the commensurability class of $S_{\alpha}$ is not comparable with $S_{\beta}$ for any $\beta \in I^{\prime}$. Therefore $I\left(S_{\alpha}\right) \stackrel{L}{\otimes} \Lambda\left(S_{\beta}\right)=0$ for every $\beta \in I^{\prime}$; so

$$
I\left(S_{\alpha}\right) \stackrel{L}{\otimes} X_{I^{\prime}}=0 .
$$

On the other hand, $I\left(S_{\alpha}\right) \stackrel{L}{\otimes} \Lambda\left(S_{\alpha}\right) \neq 0$, so $I\left(S_{\alpha}\right) \stackrel{\otimes}{\otimes}_{\Lambda}^{L} X_{I} \neq 0$. (Indeed, one can recover the set $I$ from $\left\langle X_{I}\right\rangle: I$ will consist of those indices $\beta$ for which $X_{I} \stackrel{L}{\otimes} I\left(S_{\beta}\right) \neq 0$.) This completes the proof.

Now we examine the dual picture and determine when $I(S)$ may be built from $I(T)$.

Lemma 4.8. Fix subsets $S$ and $T$ of $\mathbf{N}$.

(a) $I(S)$ is in thick $(I(T))$ if and only if $T$ is cofinite in $S$.

(b) The following are equivalent:

(1) $T \lesssim S$.

(2) $I(S)$ can be built from $I(T)$.

(3) $\langle I(S)\rangle \leqslant\langle I(T)\rangle$.

Proof. (a) If $S=T \cup\{i\}$, then we dualize the argument in Lemma 4.3(a): the BrownComenetz duals of the exact triangles in Lemma 4.1 show that $I(S)$ is in thick $(I(T))$.

Now assume that $T$ is not cofinite in $S$. As in Lemma 4.3(a), we may assume that either $T$ is not a subset of $S$ or $T \subseteq S$ with $S-T$ infinite. Since $\operatorname{Hom}_{\mathrm{D}(\Lambda(T))}^{*}(k, I(T))$ is a copy of $k$ concentrated in dimension 0 (by Lemma 3.4), then $\operatorname{Hom}_{\mathrm{D}(\Lambda(T))}^{*}\left(k,\left.X\right|_{T}\right)$ is finite-dimensional for all $X$ in thick $(I(T))$. When viewed as a $\Lambda(T)$-module, $I(S)$ splits as a direct sum:

$$
\left.\left.\left.\left.I(S)\right|_{T} \cong I(S-T)\right|_{T} \otimes I(T \cap S)\right|_{T} \cong \bigoplus I(T \cap S)\right|_{T},
$$

as in the proof of Lemma 4.3. If $S-T$ is infinite-dimensional, then the direct sum is infinite, so $\operatorname{Hom}_{\mathrm{D}(\Lambda(T))}^{0}\left(k,\left.I(S)\right|_{T}\right)$ is infinite-dimensional. Otherwise, by Lemmas 3.1 
and 3.4, we have

$$
\begin{aligned}
\operatorname{Hom}_{\mathrm{D}(\Lambda(T))}^{*}(k, I(T \cap S)) & \cong \operatorname{Hom}_{k}^{*}\left(H^{*}(k \underset{\Lambda(T)}{\stackrel{L}{\otimes}} \Lambda(T \cap S)), k\right) \\
& \cong \operatorname{Hom}_{k}^{*}\left(H^{*}(k \underset{\Lambda(U)}{\otimes} k), k\right),
\end{aligned}
$$

where $U=T-T \cap S$. The cohomology of $(k \underset{\Lambda(U)}{\stackrel{L}{\otimes}} k)$ is $\operatorname{Tor}_{*}^{\Lambda(U)}(k, k)$, which is infinitedimensional as long as $T \neq T \cap S$.

(b) As in the proof of Lemma 4.3(b), we show that if either $T=S \cup\{i\}$ or if $T \subseteq S$, then $I(S)$ can be built from $I(T)$. First assume that $T=S \cup\{i\}$. Then $I(T) \cong I(S) \otimes I(i)$, and so up to suspension, $I(T) \cong I(S) \otimes \Lambda\left(x_{i}\right)$. By Lemma $4.3, k$ may be built from $\Lambda\left(x_{i}\right)$, and so tensoring with $I(S)$, we find that $I(S)$ may be built from $I(T)$.

Now assume that $T \subseteq S$. In the special case when $T=\emptyset$, we can use the Postnikov tower for $I(S)$ to prove this; see Lemma 4.9 below. For general $T \subseteq S$, we first note that in the category $\mathrm{D}(\Lambda(S-T)), I(S-T)$ may be built from $k$, by the previous sentence. Now tensor with $I(T)$ : as $\Lambda(S)=\Lambda(S-T) \otimes \Lambda(T)$-modules, $I(S-T) \otimes I(T)$ may be built from $k \otimes I(T)$. Finally, we apply the inclusion functor from $\Lambda(S)$-modules to $\Lambda$-modules. This proves that (1) implies (2).

As explained in the proof of Lemma 4.3, (2) always implies (3).

Finally, assume that $T$ is not commensurable with any subset of $S$. This means that $U:=S \cap T$ is not cofinite in $T$. Thus $U$ is an infinite set with $U \cap S$ empty and $U \cap T$ infinite. Therefore $I(S) \stackrel{L}{\otimes} \Lambda\left(U^{c}\right) \neq 0$ while $I(T) \underset{\Lambda}{\otimes} \Lambda\left(U^{c}\right)=0$. Thus $(3)$ implies (1).

We used the following special case of the preceding lemma in its proof.

Lemma 4.9. If $S$ is any subset of $\mathbf{N}$, then $I(S)$ can be built from $k=I(\emptyset)$.

Proof. The stable homotopy theoretic version of the proof is this: use the Postnikov tower for $I(S)$. The "homotopy groups" (= homology groups in $\mathrm{D}(\Lambda)$ ) for $I(S)$ are zero except in dimension zero, and $\pi_{0}(I(S))=H^{0}(I(S))$ is the graded vector space dual of $\Lambda(S)$. When $S$ is finite, this is finite, and so the object may be built from $k$. When $S$ is infinite, this is infinite but is bounded above, so the Postnikov tower describes how to write it as a colimit of objects built from $k$.

A more explicit, homological algebra proof is as follows: first, let $S=\left\{x_{i_{1}}, x_{i_{2}}, \ldots\right\}$. Assume that $n_{i_{k}}>2$ for each $k$; the case when $n_{i_{k}}=2$ is similar but easier. Whenever $T$ is finite, $I(T)$ is a shifted copy of $\Lambda(T)$; more precisely, $I(i) \cong \Sigma^{0,-\left(n_{i}-1\right) \operatorname{deg} x_{i}} \Lambda(i)$, and when $S$ and $T$ disjoint, $I(S \cup T) \cong I(S) \otimes I(T)$, so when $T$ is finite, we have

$$
I(T) \cong \Sigma^{0,-\sum_{j \in T}\left(n_{j}-1\right) \operatorname{deg} x_{j}} \Lambda(T) .
$$

Note also that by Lemma $4.3, \Lambda(i)$ is in the thick subcategory generated by $k$, and so may be built from $k$; therefore the same is true of $I(i)$. 
Now for any $i$, the exact triangles in Lemma 4.1 give maps

$$
\begin{aligned}
k \rightarrow \Sigma^{0,-\operatorname{deg} x_{i}} M_{2} & \rightarrow \Sigma^{0,-2 \operatorname{deg} x_{i}} M_{3} \rightarrow \ldots \\
& \rightarrow \Sigma^{0,-\left(n_{i}-2\right) \operatorname{deg} x_{i}} M_{n_{i}-1} \rightarrow \Sigma^{0,-\left(n_{i}-1\right) \operatorname{deg} x_{i}} \Lambda(i),
\end{aligned}
$$

which we compose to get $k \stackrel{f_{i}}{\longrightarrow} I(i)$. Given two integers $i$ and $j$ in $\mathbf{N}$, we can form

$$
k \stackrel{f_{i}}{\longrightarrow} I(i) \stackrel{1 \otimes f_{j}}{\longrightarrow} I(i) \otimes I(j) \cong I(i, j) .
$$

In this manner, we get a composite

$$
k \rightarrow I\left(i_{1}\right) \rightarrow I\left(i_{1}, i_{2}\right) \rightarrow \ldots
$$

which displays $I(S)$ as a colimit of objects built from $k$.

We can then dualize the arguments in Corollary 4.5 and Remark 4.6 to get the following.

Corollary 4.10. For subsets $S$ and $T$ of $\mathbf{N},\langle I(S)\rangle=\langle I(T)\rangle$ if and only if $S \sim T$. Thus for subsets $S$ and $T$ of $\mathbf{N}$ with $T \lesssim S$ and $T \nsim S$, there is an uncountable antichain in the interval between $\langle I(S)\rangle$ and $\langle I(T)\rangle$.

As another application, we have another tensor product computation.

Proposition 4.11. Fix subsets $S$ and $T$ of $\mathbf{N}$. If $S$ is infinite, then $I(S) \underset{\Lambda}{\stackrel{L}{\otimes}} I(T)=0$.

For example, if $S$ is infinite, then $I(S) \stackrel{L}{\otimes} I(S)=0$ and $I(S) \underset{\Lambda}{\stackrel{L}{\otimes}} k=0$.

Proof. For fixed $S$, the collection of objects $X$ so that $I(S) \stackrel{{ }^{L}}{\otimes} X=0$ forms a localizing subcategory. By Lemma 3.6, since $S$ is infinite, this subcategory contains $\Lambda\left(S^{c}\right)$, and therefore it contains $k$ and $I(T)$, by Corollary 4.4 and Lemma 4.9. Thus $I(S) \stackrel{L}{\otimes} I(T)=0$.

If $S$ and $T$ are both finite, then $\langle I(S)\rangle=\langle I(T)\rangle=\langle k\rangle$, and so $I(S) \stackrel{L}{\otimes} I(T) \neq 0$. Thus the converse to Proposition 4.11 holds. Summarizing, we have the following.

Corollary 4.12. Fix subsets $S$ and $T$ of $\mathbf{N}$.

(a) $\Lambda(S) \stackrel{L}{\otimes} I(T)=0$ if and only if $S^{c} \cap T$ is infinite.

(b) $I(S) \stackrel{L}{\stackrel{L}{\Lambda}} I(T)=0$ if and only if $S \cup T$ is infinite.

(c) $\Lambda(S) \stackrel{L}{\otimes} \Lambda(T) \neq 0$ for all $S, T$.

Since $k=\Lambda(\emptyset)=I(\emptyset)$, we can also read off its behavior under the derived tensor product. 
Proof. Part (a) is Lemma 3.6. Part (b) is Proposition 4.11 and its converse, since $S \cup T$ is infinite if and only if either $S$ or $T$ is infinite. Part (c) follows from part (a), for instance: we know that $\langle\Lambda(T)\rangle \geqslant\langle\Lambda(\emptyset)\rangle=\langle I(\emptyset)\rangle$ for any $T$, and part (a) tells us that $\Lambda(S) \stackrel{L}{\otimes} I(\emptyset) \neq 0$.

\section{More Bousfield class calculations}

How do the Bousfield classes of the $\Lambda(S)$ 's fit together in the Bousfield lattice? We know how they are ordered; can any of them be built from the others if they are not Bousfield equivalent? The answer is no.

Write $\vee$ for the least upper bound operation on Bousfield classes. This operation is easy to understand: it is easy to verify that $\langle X\rangle \vee\langle Y\rangle=\langle X \oplus Y\rangle$, and more generally, that

$$
\bigvee_{\alpha}\left\langle X_{\alpha}\right\rangle=\left\langle\bigoplus_{\alpha} X_{\alpha}\right\rangle .
$$

Lemma 5.1. Fix subsets $S$ and $T$ of $\mathbf{N}$.

(a) Then $\langle\Lambda(S \cup T)\rangle \geqslant\langle\Lambda(S)\rangle \vee\langle\Lambda(T)\rangle$.

(b) If $S \mathbb{Z} T$ and $T \& S$, which is to say if $\langle\Lambda(S)\rangle$ and $\langle\Lambda(T)\rangle$ are not comparable in the Bousfield lattice, then this inequality is strict:

$$
\langle\Lambda(S \cup T)\rangle>\langle\Lambda(S)\rangle \vee\langle\Lambda(T)\rangle .
$$

Of course, if for example $S \lesssim T$, then $\langle\Lambda(S)\rangle \leqslant\langle\Lambda(T)\rangle$ and $S \cup T \sim T$, so we have equality: $\langle\Lambda(S \cup T)\rangle=\langle\Lambda(T)\rangle=\langle\Lambda(S)\rangle \vee\langle\Lambda(T)\rangle$.

Proof. (a) The inequality $\langle\Lambda(S \cup T)\rangle \geqslant\langle\Lambda(S)\rangle \vee\langle\Lambda(T)\rangle$ follows from Lemma 4.3(b).

(b) If $S \not T$ and $T \& S$, then $S \cup T-S$ and $S \cup T-T$ are both infinite. Therefore by Lemma 3.6 ,

$$
\Lambda(S) \stackrel{\stackrel{L}{\otimes}}{\Lambda} I(S \cup T)=0=\Lambda(T) \stackrel{L}{\stackrel{L}{\otimes}} I(S \cup T),
$$

while $\Lambda(S \cup T) \underset{\Lambda}{\stackrel{L}{\otimes}} I(S \cup T) \neq 0$. Therefore the inequality from part (a) is strict.

Dually, we have the following.

Lemma 5.2. Fix subsets $S$ and $T$ of $\mathbf{N}$.

(a) Then $\langle I(S \cap T)\rangle \geqslant\langle I(S)\rangle \vee\langle I(T)\rangle$.

(b) If $S \mathbb{Z} T$ and $T \& S$, which is to say if $\langle I(S)\rangle$ and $\langle I(T)\rangle$ are not comparable in the Bousfield lattice, then this inequality is strict: $\langle I(S \cap T)\rangle>\langle I(S)\rangle \vee\langle I(T)\rangle$.

Proof. (a) See Lemma 4.8(b).

(b) Since $S \not T$ and $T \& S$, both $S-S \cap T$ and $T-S \cap T$ are infinite. Therefore by Lemma 3.6,

$$
I(S) \stackrel{L}{\otimes} \Lambda(S \cap T)=0=I(T) \stackrel{L}{\otimes} \Lambda(S \cap T),
$$

while $I(S \cap T) \underset{\Lambda}{\stackrel{L}{\otimes}} \Lambda(S \cap T) \neq 0$. 
Notation 5.3. For any subset $S$ of $\mathbf{N}$, let $k(S)$ denote the trivial $\Lambda(S)$-module $k$. Given a partition $\mathbf{N}=A \amalg B \amalg C$, define a module $M_{A, B, C}$ over

$$
\Lambda \cong \Lambda(A) \otimes \Lambda(B) \otimes \Lambda(C)
$$

by

$$
M_{A, B, C}=\Lambda(A) \otimes k(B) \otimes I(C) .
$$

Then note, for example, that if $A$ and $A^{\prime}$ are disjoint subsets of $\mathbf{N}$, as a $\Lambda\left(A \cup A^{\prime}\right) \cong$ $\Lambda(A) \otimes \Lambda\left(A^{\prime}\right)$-module, we have $I\left(A \cup A^{\prime}\right) \cong I(A) \otimes I\left(A^{\prime}\right)$. We can compute derived tensor products involving these objects with computations like the following:

$$
\begin{aligned}
M_{A, B, C} \underset{\Lambda}{\stackrel{L}{\otimes}} \Lambda(S)= & (\Lambda(A) \otimes k(B) \otimes I(C)) \underset{\Lambda}{\stackrel{L}{\otimes}} \Lambda(S) \\
\cong & (\Lambda(A) \stackrel{L}{\otimes} \Lambda(A) \\
& \otimes(k(B) \underset{\Lambda(B)}{\stackrel{L}{\otimes}} \Lambda(B \cap S)) \\
& \otimes(I(C) \stackrel{\stackrel{L}{\otimes}}{\otimes} \Lambda(C \cap S)) .
\end{aligned}
$$

In the case of $M_{A, B, C} \stackrel{L}{\otimes} M_{S, T, U}$, there is a similar decomposition, but into nine tensor factors rather than three, yielding the following.

Lemma 5.5. Given two partitions $A \amalg B \amalg C$ and $S \amalg T \amalg U$ of $\mathbf{N}$, then

$$
M_{A, B, C} \stackrel{L}{\otimes} M_{S, T, U}=0
$$

if and only if $(C \cap T) \cup(C \cap U) \cup(B \cap U)$ is infinite.

Proof. This follows from tensor product computations like (5.4), together with Corollary 4.12 .

The results above give us the following.

Proposition 5.6. Given partitions $A \amalg B \amalg C$ and $A^{\prime} \amalg B^{\prime} \amalg C^{\prime}$ of $\mathbf{N}$, then the following are equivalent:

(1) $(A, B, C)$ is less than or equal to $\left(A^{\prime}, B^{\prime}, C^{\prime}\right)$ in the left lexicographic commensurability order; that is, either $A \lesssim A^{\prime}$ and $A \nsim A^{\prime}$, or $A \sim A^{\prime}$ and $B \lesssim B^{\prime}$.

(2) $M_{A, B, C}$ may be built from $M_{A^{\prime}, B^{\prime}, C^{\prime}}$.

(3) $\left\langle M_{A, B, C}\right\rangle \leqslant\left\langle M_{A^{\prime}, B^{\prime}, C^{\prime}}\right\rangle$.

Proof. First suppose that $A \lesssim A^{\prime}$. By Lemma 4.3, we can build $\Lambda(A)$ from $\Lambda\left(A^{\prime}\right)$. By Lemmas 4.3 and 4.8, we can build $I(C)$ from $\Lambda\left(C \cap A^{\prime}\right) \otimes k\left(C \cap B^{\prime}\right) \otimes I\left(C \cap C^{\prime}\right)$. 
That is, as $\Lambda(C)=\Lambda\left(C \cap A^{\prime}\right) \otimes \Lambda\left(C \cap B^{\prime}\right) \otimes \Lambda\left(C \cap C^{\prime}\right)$-modules, we can write

$$
I(C)=I\left(C \cap A^{\prime}\right) \otimes I\left(C \cap B^{\prime}\right) \otimes I\left(C \cap C^{\prime}\right),
$$

and then we can build this module from

$$
\Lambda\left(C \cap A^{\prime}\right) \otimes k\left(C \cap B^{\prime}\right) \otimes I\left(C \cap C^{\prime}\right) .
$$

Similarly, suppose that $A \sim A^{\prime}$ and $B \lesssim B^{\prime}$; in this case, $C^{\prime} \lesssim C$. Then we can build $I(C)$ from

$$
k\left(C \cap B^{\prime}\right) \otimes I\left(C \cap C^{\prime}\right)=k\left(C \cap B^{\prime}\right) \otimes I\left(C^{\prime}\right) .
$$

This finishes the proof that (1) implies (2). (2) implies (3) in general.

Now assume that (1) fails. That is, assume either that $A \not A^{\prime}$, or that $A \sim A^{\prime}$ and $B \not B^{\prime}$. If $A \not A^{\prime}$, then $A$ is not commensurable with any subset of $A^{\prime}$, so $A$ is not commensurable with $A \cap A^{\prime}$; this means that $A-A \cap A^{\prime}$ is infinite. Lemma 5.5 then tells us that

$$
M_{A, B, C} \stackrel{L}{\otimes} M_{C, B, A} \neq 0, \quad M_{A^{\prime}, B^{\prime}, C^{\prime}} \stackrel{L}{\otimes} M_{C, B, A}=0 .
$$

Similarly, if $A \sim B$ and $B \nsubseteq B^{\prime}$, then $B-B \cap B^{\prime}$ is infinite, as is $C^{\prime}-C \cap C^{\prime}$; indeed,

$$
B-B \cap B^{\prime} \sim C^{\prime}-C \cap C^{\prime} .
$$

Therefore we see again that

$$
M_{A, B, C} \stackrel{L}{\otimes} M_{C, B, A} \neq 0, \quad M_{A^{\prime}, B^{\prime}, C^{\prime}} \stackrel{L}{\otimes} M_{C, B, A}=0 .
$$

Thus if (1) fails, then $\left\langle M_{A, B, C}\right\rangle \nless\left\langle M_{A^{\prime}, B^{\prime}, C^{\prime}}\right\rangle$, so (3) fails. This finishes the proof.

We consider the subposet $\mathbf{m}$ of the Bousfield lattice $B$ consisting of the classes $\left\langle M_{A, B, C}\right\rangle$. Then $\mathbf{m}$ has a largest element $\hat{1}:=\langle\Lambda\rangle$ and a smallest element $\hat{0}:=\langle 0\rangle$. We show in Corollary 7.3 that $\langle I(\mathbf{N})\rangle$ is the unique minimum nonzero element in $\mathrm{B}$ (hence in $\mathbf{m}$ ): for any nonzero object $X$ in $\mathrm{D}(\Lambda)$, and in particular if $X$ happens to be of the form $X=M_{A, B, C}$, then $\langle X\rangle \geqslant\langle I(\mathbf{N})\rangle>\hat{0}$.

The poset $\mathbf{m}$ has the property that, given

$$
\langle I(\mathbf{N})\rangle \leqslant\left\langle M_{A, B, C}\right\rangle<\left\langle M_{A^{\prime}, B^{\prime}, C^{\prime}}\right\rangle \leqslant \hat{1}
$$

the interval

$$
\left[\left\langle M_{A, B, C}\right\rangle,\left\langle M_{A^{\prime}, B^{\prime}, C^{\prime}}\right\rangle\right]
$$

in $\mathbf{m}$ contains an uncountable antichain; see Remark 4.6 and Corollary 4.5.

Now define $\mathbf{M}$ to be the lattice obtained by closing $\mathbf{m}$ under arbitrary joins in $\mathbf{B}$, so the elements of $\mathbf{M}$ are the Bousfield classes of direct sums of the objects $M_{A, B, C}$. By Theorem 4.7, $\mathbf{M}$ has cardinality at least $2^{2^{\aleph_{0}}}$.

We end this section with a few questions.

Question 5.7. Is $B=\mathbf{M}$ ? That is, is every object in $\mathrm{D}(\Lambda)$ Bousfield equivalent to an object of the form

$$
\bigoplus_{\alpha} M_{A_{\alpha}, B_{\alpha}, C_{\alpha}} ?
$$


Note that a positive answer would provide headway toward solving [HP99, Conjecture 9.1], that every localizing subcategory in $\mathrm{D}(\Lambda)$ is a Bousfield class: according to $[\mathbf{H P 9 9}$, Proposition 9.2], the conjecture is equivalent to the statement that $X$ can be built from $Y$ if and only if $\langle X\rangle \leqslant\langle Y\rangle$, for all $X$ and $Y$. Results like Proposition 5.6 seem like progress in this direction.

On the other hand, a positive answer to this question may very well be too much to ask. Here is a variant.

Question 5.8. Let $R$ be a commutative ring. Is every object in $\mathrm{D}(R)$ Bousfield equivalent to an $R$-module?

This is true if $R$ is noetherian, by Neeman's work [Nee92]. In general, one might guess that any object $X$ is Bousfield equivalent to the sum of its homology groups, $\bigoplus_{i} H^{i}(X)$.

Finally, we have the following.

Question 5.9. Let $R$ be a commutative ring. Does the Bousfield lattice for $\mathrm{D}(R)$ form a set?

The answer is "yes" if $R$ is noetherian or countable: in the noetherian case, Neeman's work [Nee92] establishes a bijection between the Bousfield lattice and the lattice of subsets of Spec $R$. In the countable case, $\mathrm{D}(R)$ is a Brown category see the discussion just before Notation 2.1 - and so Ohkawa's theorem holds, as explained in [DP01].

\section{Objects with large tensor-nilpotence height}

If $X$ is an object in $\mathrm{D}(\Lambda)$, write $X^{(n)}$ for the $n$-fold derived tensor product of $X$ with itself.

\section{Theorem 6.1.}

(a) For each $n \geqslant 1$, there is an object $X$ so that $X^{(n)}$ is nonzero while $X^{(n+1)}$ is zero.

(b) There is an object $Y$ for which

$$
\langle Y\rangle>\left\langle Y^{(2)}\right\rangle>\left\langle Y^{(3)}\right\rangle>\cdots
$$

Proof. (The idea for this proof arose from a conversation with James Zhang.)

(a) Fix an integer $n \geqslant 1$ and choose subsets $S_{i} \subseteq \mathbf{N}, 1 \leqslant i \leqslant n$, so that each $S_{i}$ is infinite and the $S_{i}$ 's are pairwise disjoint. Define a $\Lambda$-module $X$ by

$$
X=\bigoplus_{i} I\left(S_{i}\right) \otimes \Lambda\left(S_{i}^{c}\right)=\bigoplus_{i} M_{S_{i}^{c}, \emptyset, S_{i}}
$$

with notation as in 5.3. For disjoint subsets $S, T \subseteq \mathbf{N}$, we have

$$
\left(I(S) \otimes \Lambda\left(S^{c}\right)\right) \underset{\Lambda}{\stackrel{L}{Q}}\left(I(T) \otimes \Lambda\left(T^{c}\right)\right)=I(S \cup T) \otimes \Lambda\left((S \cup T)^{c}\right),
$$

and if $S$ is infinite, then

$$
\left(I(S) \otimes \Lambda\left(S^{c}\right)\right) \stackrel{\perp}{\perp}_{\Lambda}^{L}\left(I(S) \otimes \Lambda\left(S^{c}\right)\right)=0 .
$$


Therefore we have

$$
\begin{aligned}
X \stackrel{L}{\otimes} X & =\bigoplus_{1 \leqslant i<j \leqslant n} I\left(S_{i} \cup S_{j}\right) \otimes \Lambda\left(\left(S_{i} \cup S_{j}\right)^{c}\right), \\
X^{(m)} & =\bigoplus_{1 \leqslant i_{1}<\cdots<i_{m} \leqslant n} I\left(S_{i_{1}} \cup \cdots \cup S_{i_{m}}\right) \otimes \Lambda\left(\left(S_{i_{1}} \cup \cdots \cup S_{i_{m}}\right)^{c}\right), \\
X^{(n)} & =I\left(S_{1} \cup \cdots \cup S_{n}\right) \neq 0, \\
X^{(n+1)} & =0 .
\end{aligned}
$$

This completes the proof of part (a).

The proof of part (b) is similar. As in the proof of Corollary 4.5, for each prime number $p$, we let $P_{p}$ be the set of powers of $p$. Then define $Y$ by

$$
Y=\bigoplus_{p} I\left(P_{p}\right) \otimes \Lambda\left(P_{p}^{c}\right)
$$

Then, computing as in part (a), we have

$$
Y^{(m)}=\bigoplus_{p_{1}<\cdots<p_{m}} I\left(P_{p_{1}} \cup \cdots \cup P_{p_{m}}\right) \otimes \Lambda\left(\left(P_{p_{1}} \cup \cdots \cup P_{p_{m}}\right)^{c}\right) .
$$

List the prime numbers as $p(1), p(2), p(3), \ldots$, and let

$$
U=P_{p(m)} \cup P_{p(m+1)} \cup P_{p(m+2)} \cup \cdots .
$$

Then $\Lambda\left(U^{c}\right){ }_{\Lambda}^{L} Y^{(m)}$ is zero by Lemma 3.6, because $U^{c}$ has an infinite intersection with any $m$-fold union of the $P_{p}$ 's. On the other hand, $\Lambda\left(U^{c}\right) \stackrel{L}{Q}_{\Lambda}^{(m-1)}$ is nonzero, because $U^{c} \cap\left(P_{p(1)} \cup \cdots \cup P_{p(m-1)}\right)$ is empty.

As in the usual stable homotopy category [Bou79, HP99], we define the distributive lattice DL to be the sub-poset of the Bousfield lattice B consisting of classes $\langle X\rangle$ with $\langle X\rangle=\langle X \stackrel{L}{\otimes} \underset{\Lambda}{\otimes}\rangle\rangle$; this does not contain $\langle I(S)\rangle$ if $S$ is infinite, but it does contain the classes $\langle\Lambda(S)\rangle$, because each $\Lambda(S)$ is a ring object.

The methods in [HP99, Section 3] apply here to give an epimorphism of lattices $r: \mathrm{B} \rightarrow \mathrm{DL}$, right adjoint to the inclusion $\mathrm{DL} \rightarrow \mathrm{B}$; furthermore, the map $r$ factors through an epimorphism

$$
r^{\prime}: \mathrm{B} / J \rightarrow \mathrm{DL}
$$

where $J$ is the ideal in B of Bousfield classes less than $\langle k\rangle$. The "retract conjecture" [HP99, Conjecture 3.12] says that $r^{\prime}$ is an isomorphism.

Corollary 6.2. The retract conjecture fails in $\mathrm{D}(\Lambda)$.

Proof. According to [HP99, Proposition 3.13(e)], the retract conjecture implies that every object $E$ in $\mathrm{D}(\Lambda)$ satisfies

$$
\langle E \underset{\Lambda}{\otimes} E\rangle=\langle E \underset{\Lambda}{\stackrel{L}{\otimes}} \underset{\Lambda}{\stackrel{L}{\otimes}} E\rangle
$$

The theorem says that this is not true. 


\section{Minimality of $\langle I(\mathbf{N})\rangle$}

In this section, we prove the following theorem.

Theorem 7.1. Fix objects $E$ and $X$ in $\mathrm{D}(\Lambda)$. If $E \neq 0$ and $X \stackrel{L}{\otimes} E=0$, then we have $\operatorname{RHom}_{\Lambda}(X, \Lambda)=0$ and $\operatorname{Hom}_{\mathrm{D}(\Lambda)}(X, \Lambda)=0$.

A stable homotopy theorist would translate this as, "The object $\Lambda$ in $\mathrm{D}(\Lambda)$ is local with respect to every nonzero homology theory." Thus the theorem says that every nonzero object has a finite local, namely $\Lambda$. This verifies the "Dichotomy conjecture" from [HP99, Conjecture 7.5] in the category $\mathrm{D}(\Lambda)$. (Since no object can have both a finite local and a finite acyclic, this also implies that no nonzero object has a finite acyclic; this also follows from Corollary 8.3(b) below.)

Theorem 7.1 has a number of useful consequences. Taking the contrapositive gives the following.

Corollary 7.2. If $\operatorname{RHom}_{\Lambda}(X, \Lambda) \neq 0$, then $X$ is Bousfield equivalent to $\Lambda$.

Corollary 7.3. $I(\mathbf{N})$ is the minimum nonzero Bousfield class: that is, for every nonzero object $E$, we have $\langle E\rangle \geqslant\langle I(\mathbf{N})\rangle$.

Proof. Fix a nonzero object $E$ in $\mathrm{D}(\Lambda)$ and suppose that $Y \underset{\Lambda}{\otimes} E=0$. Then Theo-

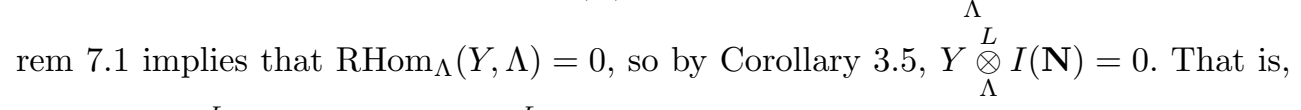
whenever $Y \stackrel{L}{\stackrel{L}{\otimes}} E=0$, we have $Y \stackrel{L}{\stackrel{L}{Q}} I(\mathbf{N})=0$. Thus $\langle E\rangle \geqslant\langle I(\mathbf{N})\rangle$.

As in the usual stable homotopy category, we define the Boolean algebra BA to be the set of complemented Bousfield classes: those classes $\langle X\rangle$ for which there is a $Y$ with

$$
\langle X \underset{\Lambda}{\stackrel{L}{\otimes}} Y\rangle=0, \quad\langle X \oplus Y\rangle=\Lambda .
$$

In any stable homotopy category, we have the inclusion BA $\subseteq$ DL; see [Bou79] and [HP99] for more about BA, DL, and B.

Corollary 7.4. In the Bousfield lattice for the category $\mathrm{D}(\Lambda)$, BA is trivial: the only complemented classes are $\langle 0\rangle$ and $\langle\Lambda\rangle$.

Proof. Fix $\langle X\rangle$ not equal to $\langle 0\rangle$ or $\langle\Lambda\rangle$. Suppose that $Y \neq 0$ and $X \stackrel{L}{\otimes} Y=0$. Then by Corollary 7.3, $\langle X\rangle \geqslant\langle I(\mathbf{N})\rangle$ and $\langle Y\rangle \geqslant\langle I(\mathbf{N})\rangle$. Thus $(X \oplus Y) \underset{\Lambda}{\otimes} I(\mathbf{N})=0$, so there is no object $Y$ so that $X \stackrel{L}{\otimes} Y=0$ and $\langle X \oplus Y\rangle=\langle\Lambda\rangle$.

The telescope conjecture in a general stable homotopy category says that every smashing localization is a finite localization; see [HPS97, 3.3.8].

Corollary 7.5. The telescope conjecture holds in $\mathrm{D}(\Lambda)$. 
Proof. Suppose that $\langle E\rangle$ is a nonzero smashing Bousfield class. From [Rav84, 1.31] or [HP99, 4.5], we have that $\langle E\rangle$ must be complemented, and so by Corollary 7.4, we have $\langle E\rangle=\langle\Lambda\rangle$. Localization with respect to $\Lambda$ is the identity functor, which is trivially a finite localization.

We need a few lemmas before we prove the theorem.

Fix a ring $R$ and a subring $S$; then for any object $Y$ in $\mathrm{D}(R)$, write $\left.Y\right|_{S}$ for the restriction of $Y$ to $S$.

Lemma 7.6. Let $S$ be a graded commutative ring, and let $R=S[x] /\left(x^{n}\right)$. Assume that $R$ is graded commutative; that is, if $n>2$, assume that $\operatorname{deg} x$ is even. For any object $X$ in $\mathrm{D}(R)$, one can build $\left.R \underset{S}{\stackrel{L}{\otimes}} X\right|_{S}$ from $X$.

Proof. The proof goes like this: first we claim that one can build $R \underset{S}{\otimes} R=\left.R \underset{S}{\otimes} R\right|_{S}$ from $R$. Given this, we apply $-\stackrel{L}{\otimes} X$ to deduce that one can build $\left.R \stackrel{L}{\otimes} X\right|_{S}$ from $X$. There is an important technical point, though: while it is clear that one can build $R \underset{S}{\otimes} R$ from $R$ as, say, a right $R$-module (since one can build anything in the derived category of right $R$-modules from $R$ ), then after tensoring with $X$, we would only know that we could build $R \underset{S}{\otimes} X$ from $X$ as a left $S$-module, not as a left $R$-module.

So the lemma is equivalent to this claim: one can build $R \underset{S}{\otimes} R$ from $R$ as an $(R, R)$-bimodule. Of course, $(R, R)$-bimodules are the same as modules over

$$
R \underset{S}{\otimes} R^{\mathrm{op}} \cong R \underset{S}{\otimes} R \cong S[x, y] /\left(x^{n}, y^{n}\right) .
$$

Thus we make $S[x] /\left(x^{n}\right)$ into a module over $S[x, y] /\left(x^{n}, y^{n}\right)$ by having both $x$ and $y$ act as multiplication by $x$; then we want to show that we can build $S[x, y] /\left(x^{n}, y^{n}\right)$ from $S[x] /\left(x^{n}\right)$. We do this in two steps.

First, we build $S$ from $S[x] /\left(x^{n}\right)$ in $\mathrm{D}\left(S[x, y] /\left(x^{n}, y^{n}\right)\right)$. We do this by taking a free resolution of $S$ as an $S[x] /\left(x^{n}\right)$-module, treating the result as a chain complex of (non-free) modules over $S[x, y] /\left(x^{n}, y^{n}\right)$, and then taking successive truncations as in the proof of Lemma 4.3. That is, $S$ is the colimit of the chain complexes

$$
0 \rightarrow P_{m} \rightarrow \cdots \rightarrow P_{0} \rightarrow 0
$$

where each $P_{i}$ is a shifted copy of $S[x] /\left(x^{n}\right)$. Each such chain complex can be built from $S[x] /\left(x^{n}\right)$, and hence so can $S$.

Second, using the fact that $S[x, y] /\left(x^{n}, y^{n}\right)$ is free of finite rank as an $S$-module, we can build it from $S$. Since we can build $S$ from $S[x] /\left(x^{n}\right)$, this completes the proof.

Lemma 7.7. Let $T$ be a subset of $\mathbf{N}$ with $\mathbf{N}-T$ finite. Fix an object $Y$ in $\mathrm{D}(\Lambda)$. If the free module $\Lambda(T)$ is a summand of $\left.Y\right|_{\Lambda(T)}$, then $\Lambda$ may be built from $Y$, so $\langle Y\rangle=\langle\Lambda\rangle$.

Proof. First assume that $\Lambda$ may be built from $Y$; then $\langle Y\rangle \geqslant\langle\Lambda\rangle$; on the other hand, $\langle Y\rangle \leqslant\langle\Lambda\rangle$ for any $Y$, so we see that $\langle Y\rangle=\langle\Lambda\rangle$. 
So we need to verify that $\Lambda$ may be built from $Y$. Write $\mathbf{N}-T=\left\{x_{i_{1}}, \ldots, x_{i_{n}}\right\}$. For each $j, 1 \leqslant j \leqslant n$, let

$$
\Lambda_{j}=\Lambda\left(T \cup\left\{x_{i_{1}}, \ldots, x_{i_{j}}\right\}\right)
$$

and let $\Lambda_{0}=\Lambda(T)$. Note that $\Lambda_{n}=\Lambda$. Then for each $j \leqslant n$, there is an algebra isomorphism

$$
\Lambda_{j} \cong \Lambda_{j-1}\left[x_{j}\right] /\left(x_{j}^{n_{j}}\right),
$$

so we may apply the previous lemma to conclude that we may build $\left.\Lambda_{j} \stackrel{L}{\otimes} Y \Lambda_{j-1}\right|_{\Lambda_{j-1}}$ from $\left.Y\right|_{\Lambda_{j}}$ for each $j$. Since $\Lambda_{0}$ is a summand of $\left.Y\right|_{\Lambda_{0}}$, we may build $\Lambda_{1} \underset{\Lambda_{0}}{\otimes} \Lambda_{0} \cong \Lambda_{1}$ from $\left.Y\right|_{\Lambda_{1}}$. Inductively, we see that we may build $\Lambda_{j}$ from $\left.Y\right|_{\Lambda_{j}}$ for each $j$, and in particular when $j=n$, we may build $\Lambda_{n}=\Lambda$ from $Y$.

Proof of Theorem 7.1. Fix an object $X$ of $\mathrm{D}(\Lambda)$ and suppose that $\operatorname{Hom}_{\mathrm{D}(\Lambda)}(X, \Lambda)$ $\neq 0$. We want to show that if $X \stackrel{L}{\otimes} E=0$, then $E=0$; that is, we want to show that $X$ is Bousfield equivalent to the sphere object $\Lambda$.

So suppose that $f: X \rightarrow \Lambda$ is a nonzero map. Then one can check that it induces a nonzero map on homology: $H(f): H^{0}(X) \rightarrow \Lambda$ is nonzero. This is a map of $\Lambda$-modules. If $H(f)(y) \neq 0$, then the element $y \in H^{0}(X)$ supports free actions by all but finitely many of the $x_{i}$ 's, because this is true for its target in $\Lambda$. That is, there is a set $T$, cofinite in $\mathbf{N}$, so that in the homology of the restriction of $X$ to $\Lambda(T)$, $y$ generates a free module of rank one. Therefore this free module will split off of $\left.X\right|_{\Lambda(T)}$. By Lemma 7.7 , this means that $\langle X\rangle=\langle\Lambda\rangle$, as desired.

\section{Nilpotence and small objects}

In this section, we discuss versions of some stable homotopy results in the category $\mathrm{D}(\Lambda)$. In particular, we show that the object $k$ detects nilpotence, in the language of [HS98, Hop87]. We use this to classify the thick subcategories of small objects, and also to prove a version of the periodicity theorem [HS98, Hop87].

The homology of an object $X$ in the derived category $\mathrm{D}(\Lambda)$ is $\operatorname{Hom}_{\mathrm{D}(\Lambda)}^{*}(\Lambda, X)$, which is not the same as $k_{*} M$. Indeed, if $M$ is a $\Lambda$-module, then

$$
k_{*} M:=\operatorname{Hom}_{\mathrm{D}(\Lambda)}^{*}(\Lambda, k \underset{\Lambda}{\stackrel{L}{\otimes}} M) \cong H_{*}(k \stackrel{L}{\stackrel{L}{\otimes}} M) \cong \operatorname{Tor}_{*}^{\Lambda}(k, M) .
$$

In order to state the nilpotence theorem, we need to recall a few definitions.

\section{Definition 8.1.}

(a) First, $E$ is a ring object in $\mathrm{D}(\Lambda)$ if there is a "multiplication" map $\mu: E \stackrel{L}{\otimes} E \rightarrow E$ and a "unit" map $\eta: \Lambda \rightarrow E$ in $\mathrm{D}(\Lambda)$ so that $\mu \circ(\eta \otimes 1)=1=\mu \circ(1 \otimes \eta)$. If $E$ is a ring object, then $\operatorname{Hom}_{\mathrm{D}(\Lambda)}^{*}(\Lambda, E)$ is a (graded) ring: given two maps 
$f, g: \Lambda \rightarrow E$, their product is the composite

$$
\Lambda \stackrel{\cong}{\longrightarrow} \Lambda \stackrel{L}{\otimes} \Lambda \stackrel{f \otimes g}{\longrightarrow} E \stackrel{L}{\otimes} E \stackrel{\mu}{\longrightarrow} E .
$$

Similarly, since $k$ is a ring object, so is $k \underset{\Lambda}{\otimes} E$, and so $k_{*} E=\operatorname{Hom}_{\mathrm{D}(\Lambda)}^{*}(\Lambda, k \underset{\Lambda}{\stackrel{L}{\otimes}} E)$ is a graded ring.

(b) An object $F$ in $\mathrm{D}(\Lambda)$ is small if the natural map

$$
\bigoplus_{\alpha} \operatorname{Hom}_{\mathrm{D}(\Lambda)}\left(F, X_{\alpha}\right) \rightarrow \operatorname{Hom}_{\mathrm{D}(\Lambda)}\left(F, \bigoplus_{\alpha} X_{\alpha}\right)
$$

is an isomorphism for any set of objects $\left\{X_{\alpha}\right\}$.

(c) A map $g: F \rightarrow F$ is nilpotent if some composite $g \circ \cdots \circ g$ is zero. A map $f: F \rightarrow X$ is tensor-nilpotent if for some $n \geqslant 1$, the map

$$
f^{\otimes n}: \underbrace{F \stackrel{L}{\otimes} \cdots \stackrel{L}{\otimes} F}_{n} \rightarrow \underbrace{X \stackrel{L}{\otimes} \cdots \stackrel{L}{\otimes} X}_{n}
$$

is zero.

Theorem 8.2 (Nilpotence theorem). The field object $k=\Lambda(\emptyset)$ detects nilpotence:

(a) For any ring object $E$ and $\alpha: \Lambda \rightarrow E, \alpha$ is nilpotent if and only if $k_{*} \alpha$ is nilpotent.

(b) For any small object $F$ and self-map $g: F \rightarrow F, g$ is nilpotent if and only if $k_{*} g$ is nilpotent.

(c) For any small object $F$, any object $X$, and any map $f: F \rightarrow X$, the map $f$ is tensor-nilpotent if and only if $k_{*} f$ is zero.

Proof. Since $\Lambda$ is the unit of the derived tensor product in $\mathrm{D}(\Lambda)$, it plays the role of the sphere object. Therefore, as in the proof of [HS98, Theorem 3], the proof boils down to this situation: $f: \Lambda \rightarrow X$ is a map, and $T$ is the following telescope:

$$
T=\lim _{\longrightarrow}\left(\Lambda \stackrel{f}{\rightarrow} X \stackrel{f \otimes 1_{X}}{\longrightarrow} X \stackrel{L}{\otimes} X \stackrel{f \otimes 1_{X} \otimes 1_{X}}{\longrightarrow} X \underset{\Lambda}{\otimes} X \underset{\Lambda}{\stackrel{L}{\otimes}} X \rightarrow \cdots\right) .
$$

We assume that $k_{*} f=0$, and we want to show that the $m$ th derived tensor power $f^{(m)}$ of $f$ is zero for $m \gg 0$, or equivalently, that $T=0$. From Lemma 4.3, we see that for any $n \geqslant 1$,

$$
\langle\Lambda\rangle=\langle\Lambda(n, n+1, \ldots)\rangle .
$$

Since $k=\lim _{\longrightarrow} \Lambda(n, n+1, \ldots)$, the map

$$
\Lambda \rightarrow \lim _{\longrightarrow} \Lambda(n, n+1, \ldots) \stackrel{L}{\stackrel{L}{\Lambda}} T
$$

is null. Thus since $\Lambda$ is small in the category $\mathrm{D}(\Lambda)$, we see that for some $n \geqslant 1$,

$$
\Lambda \rightarrow \Lambda(n, n+1, \ldots) \stackrel{L}{\otimes} T
$$


is null, which means that

$$
\Lambda(n, n+1, \ldots) \underset{\Lambda}{\stackrel{L}{\otimes}} T=0,
$$

and hence $T=\Lambda \stackrel{L}{\otimes} T=0$, as desired.

It is a standard result that the subcategory of small objects in the derived category of a commutative ring $R$ is precisely the thick subcategory (Definition 4.2) generated by $R$. Also, whenever one has field objects which detect nilpotence, they determine the thick subcategories of the category of small objects; see [HPS97, Corollary 5.2.3]. Thus since $k$ is a field object, Theorem 8.2 gives the following.

Corollary 8.3 (Thick subcategory theorem). Consider the category $\mathrm{F}=$ thick $(\Lambda)$ of small objects of $\mathrm{D}(\Lambda)$.

(a) Every thick subcategory of $\mathrm{F}$ is trivial: the only thick subcategories are $\{0\}$ and $\mathrm{F}$.

(b) Every nonzero small object in $\mathrm{D}(\Lambda)$ is Bousfield equivalent to the sphere object $\Lambda$.

Part (a) also follows from a result of Thomason [Tho97, Theorem 3.15].

Proposition 8.4 (Nishida's theorem, periodicity theorem). For any small object $F$ in $\mathrm{D}(\Lambda)$, any self-map $g: F \rightarrow F$ of nonzero degree is nilpotent.

Proof. Fix a map $g: \Sigma^{a, b} F \rightarrow F$ with $(a, b) \neq(0,0)$. Since $k_{*} \Lambda$ consists of a single copy of $k$ in degree $(0,0)$, we see that for any object $F$ in thick $(\Lambda), k_{*} F$ is finitedimensional as a vector space over $k$. Thus for degree reasons, $k_{*} g$ must be nilpotent, so Theorem 8.2 says that $g$ is nilpotent.

\section{References}

[BC76] E. H. Brown and M. Comenetz, Pontrjagin duality for generalized homology and cohomology theories, Amer. J. Math. 98(1) (1976), 1-27. MR 53 \#9196

[Bou79] A. K. Bousfield, The Boolean algebra of spectra, Comment. Math. Helv. 54(3) (1979), 368-377. MR 81a:55015

[CE56] H. Cartan and S. Eilenberg, Homological algebra, Princeton University Press, Princeton, N. J., 1956.

[DP01] W. G. Dwyer and J. H. Palmieri, Ohkawa's theorem: there is a set of Bousfield classes, Proc. Amer. Math. Soc. 129(3) (2001), 881-886. MR 1712921

[Hop87] M. J. Hopkins, Global methods in homotopy theory, in Homotopy theory (Durham, 1985) (J. D. S. Jones and E. Rees, eds.), London Math. Soc. Lecture Note Series 117, 73-96, Cambridge Univ. Press, New York, 1987. 
[HP99] M. Hovey and J. H. Palmieri, The structure of the Bousfield lattice, in Homotopy invariant algebraic structures (J.-P. Meyer, J. Morava, and W. S. Wilson, eds.), Contemp. Math. 239, Amer. Math. Soc., Providence, RI, 1999, 175-196.

[HPS97] M. Hovey, J. H. Palmieri, and N. P. Strickland, Axiomatic stable homotopy theory, Mem. Amer. Math. Soc. 128 (1997), no. 610. MR 98a:55017

[HS98] M. J. Hopkins and J. H. Smith, Nilpotence and stable homotopy theory II, Ann. of Math. (2) 148(1) (1998), 1-49.

[Mar83] H. R. Margolis, Spectra and the Steenrod algebra. Modules over the Steenrod algebra and the stable homotopy category, North-Holland Publishing Co., New York, 1983.

[Nee92] A. Neeman, The chromatic tower for D(R), Topology 31(3) (1992), 519532.

[Nee00] A. Neeman, Oddball Bousfield classes, Topology 39(5) (2000), 931-935. MR 1763956 (2001c:18007)

[Rav84] D. C. Ravenel, Localization with respect to certain periodic homology theories, Amer. J. Math. 106(2) (1984), 351-414.

[Tho97] R. W. Thomason, The classification of triangulated subcategories, Compositio Math. 105(1) (1997), 1-27.

W. G. Dwyer dwyer.1@nd.edu

Department of Mathematics, University of Notre Dame, Notre Dame, IN 46556, USA

J. H. Palmieri palmieri@math.washington.edu

Department of Mathematics, Box 354350, University of Washington, Seattle, WA 98195, USA 